NASA/CR-1998-208448

ICASE Report No. 98-28

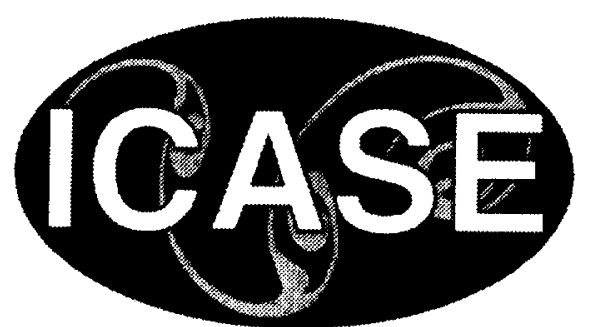

\title{
Energy Spectra of Strongly Stratified and Rotating Turbulence
}

\author{
Alex Mahalov and Basil Nicolaenko \\ Arizona State University, Tempe, Arizona
}

Ye Zhou

ICASE, Hampton, Virginia

and

IBM, Yorktown Heights, New York

Institute for Computer Applications in Science and Engineering

NASA Langley Research Center

Hampton, VA

Operated by Universities Space Research Association

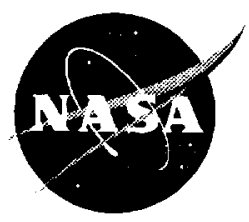

National Aeronautics and

Space Administration

Langley Research Center

Hampton, Virginia 23681-2199 
Available from the following:

NASA Center for AeroSpace Information (CASI) 7121 Standard Drive

Hanover, MD 21076-1320

(301) 621-0390
National Technical Information Service (NTIS) 5285 Port Royal Road

Springfield, VA 22161-2171

(703) $487-4650$ 


\title{
ENERGY SPECTRA OF STRONGLY STRATIFIED AND ROTATING TURBULENCE*
}

\author{
ALEX MAHALOV ${ }^{\dagger}$, BASIL NICOLAENKO ${ }^{\ddagger}$, AND YE ZHOU $§$
}

\begin{abstract}
Turbulence under strong stratification and rotation is usually characterized as quasi-two dimensional turbulence. We develop a "quasi-two dimensional" energy spectrum which changes smoothly between the Kolmogorov $-5 / 3$ law (no stratification), the -2 scalings of Zhou for the case of strong rotation, as well as the -2 scalings for the case of strong rotation and stratification. For strongly stratified turbulence, the model may give the -2 scaling predicted by Herring; and the $-5 / 3$ scaling indicated by some mesoscale observations.
\end{abstract}

Key words. stratification, rotation, turbulence modeling

Subject classification. Fluid Mechanics

1. Introduction. The important applications of turbulence under strong stratification and rotation in geophysics and enginecring are well documented (Pedlosky [1], Hopfinger [2], Rodi [3], Fernando and Hunt [4], Rubinstein and Zhou [5]). As an example, large-scale flows in oceans and earth's atmosphere are known as almost two dimensional, but not exactly so. Typically, one characterizes this type of flows as quasi-two dimensional turbulence. In this note, we discuss the development of the spectra for turbulence subject to strong (stable) stratification and rotation.

We define the asymptotic regimes of geophysical dynamics in terms of the following non-dimensional parameters. Let $H$ be the vertical (spectral) length scale, $L$ be the horizontal length scalc, and $U_{h}$ be a characteristic horizontal velocity scale. Then the spectral aspect ratio can be defined as $a=H / L$. We define Froude number based on horizontal and vertical scales:

$$
F_{h}=U_{h} / L N_{0} \equiv 1 / N, F_{v}=U_{h} / H N_{0}=F_{h} / a .
$$

The classical Rossby and anisotropic Rossby numbers are defined as follows

$$
R o=U_{h} / L f_{0} \equiv 1 / f, R o_{a}=R o \times a .
$$

Here $N_{0}$ is the Brunt-Väisälä frequency for constant stratification gradient and $f_{0}=2 \Omega_{0}$ is the Coriolis parameter ( $\Omega_{0}$ is the frequency of background rotation); the vertical axis is taken to be aligned with the axis of rotation and the mean stratification gradient. The governing flow equations are three-dimensional (3D) Euler-Boussinesq equations for rotating stratified fluids with zero-flux boundary conditions in the vertical direction. Such boundary conditions imply zero tangential stress on the vertical boundaries.

The Burger number characterizes relative importance of the effects of rotation and stratification (McWilliams [10]):

$$
B u=R o_{a}^{2} / F_{h}^{2} \equiv R o^{2} / F_{v}^{2} \equiv N_{0}^{2} a^{2} / f_{0}^{2}
$$

\footnotetext{
*This research was supported by the AFOSR (grant F49620-93-1-0172), the NSF Environmental Geochemistry and Biochemistry Initiative (grant 97-08452), the ASU Center for Environmental Fluid Dynamics, and by the National Aeronautics and Space Administration under NASA Contract No. NAS1-97046 while the third author was in residence at the Institute for Computer Applications in Science and Engineering (ICASE), NASA Langley Research Center, Hampton, VA 23681-2199.

†Department of Mathematics, Arizona State University, Tempe, AZ 85287 .

$\ddagger$ Department of Mathematics, Arizona State University, Tempe, AZ 85287.

$\S$ Institute for Computer Applications in Science and Engineering, NASA Langley Research Center, Hampton, VA 23681 and IBM Research Division, T.J. Watson Research Center, P.O. Box, 218, Yorktown Heights, NY 10598.
} 


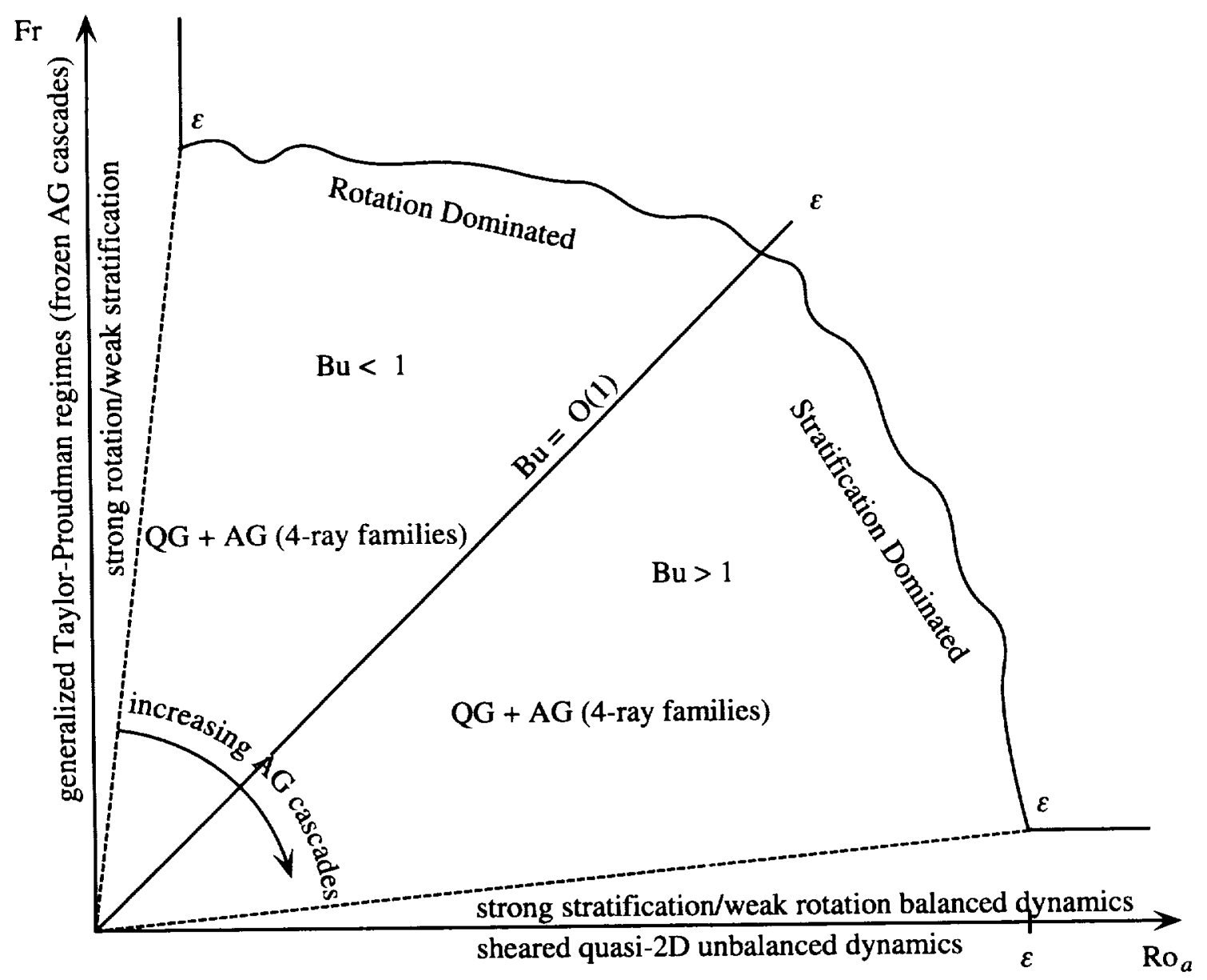

Fig. 1. Geophysical Dynamics: the global picture for small Froude or small Rossby regimes.

FIG. 1.

with $B u<<1$ corresponding to rotation-dominated and $B u>>1$ corresponding to stratification-dominated flows. Also, a measure of the relative importance of (stable) stratification versus rotation is the internal radius of deformation $\Lambda$. The internal (Rossby) radius of deformation $\Lambda$ is defined as

$$
\Lambda=N_{0} H / f_{0},
$$

so that $B u=(\Lambda / L)^{2}$. When $L>>\Lambda$, the flow is organized in quasi-2D vertical columns and when $L<<\Lambda$, the flow is organized in thin horizontal layers with a strong vertical variability.

From the mathematical analysis (Babin et al., [6-9]), we cor.structed Fig. 1 to illustrate the global picture of geophysical dynamics at small Froude and/or small Rossby regimes. Since we are not taking $a \rightarrow 0$, either $F_{h}$ or $F_{v}$ can be used in description of asymptotic regimes. Then $F r$ denotes either of these numbers.

When only strong rotation exists (Fig. 1, vertical axis), any solution of the initial value problem for 3D Euler-Boussinesq/Navier-Stokes system can be split into two parts. The first component is a solution of twodimensional (2D) barotropic Euler-Boussinesq/Navier-Stokes system with vertically averaged initial data. The dynamics of the second part which describes vertical variability is called ageostrophic in this limiting context. In this asymptotic regime, it is exactly solved in terms of $2 \mathrm{D}$ dynamics of vertically averaged fields. The error of the splitting is at the order of the anisotropic Rossby number (defined by Eq. (2)), a very 
small number in many situations (Babin et al., [6], [8-9]). Energy cascades for the ageostrophic modes are completely frozen in the vertical direction and the ageostrophic dynamics is pure phase turbulence. In pure phase turbulence, the amplitudes of the ageostrophic modes remain constant in absolute values; turbulent dynamics are restricted to the phases of the ageostrophic modes. The ageostrophic field is phase locked to phases associated with vertically averaged vertical vorticity and vertical velocity which are advected by $2 \mathrm{D}$ turbulence of vertically averaged fields. There is no slaving of the amplitudes of ageostrophic modes by the 2D turbulence, only phase locking.

When stratification is present, the cascades of ageostrophic modes (AG) become 'unfrozen'. As stratification increases, the direct cascade of ageostrophic energy from large scales to small scales increases (Babin et al., [8]). When both rotation and stratification effects are of the same order of magnitude - the situation called Burger one regime (McWilliams [10]), Babin et al. [7-8] established the splitting between 3D quasigeostrophic (QG) and reduced ageostrophic fields using the Craya-Herring cyclic bases (Lesieur, [11]). In these bases the ageostrophic modes are characterized in terms of the divergent velocity potential (horizontal divergence) and the geostrophic departure/thermal wind unbalance (e.g. [8]). The QG modes inverse transfer the vortical (rotational) energy upscales. On the other hand, direct energy cascades of the AG ficld provide a mechanism for nonlinear geostrophic adjustment. This is fundamentally different from the rotation dominated regimes where AG cascades are frozen. The nonlinear geostrophic adjustment mechanism is indeed the capacity of the $A G$ dynamics to transfer energy to smaller scales and eventually dissipate its inertio-gravitational energy (Farge and Sadourny, [12]). Direct cascades of energy of the ageostrophic modes indicate that the observed $-5 / 3$ power-law is the spectrum of internal gravity waves with direct energy cascade to large wavenumbers (small scales).

2. Model Development. In order to infer the form of the incrtial-range spectrum $E(k)$ for different asymptotic regimes, it is necessary to estimate the magnitude for the triple velocity correlations. In general, $\tau_{3}$, the time scale for the decay of the triple correlations responsible for inducing turbulent spectral transfer, may depend on any relevant turbulence parameters (Kraichnan [13], Zhou [14]). When energy is conserved by the nonlinear interaction and a local cascade has been assumed, the energy flux, which equals to the dissipation rate $\epsilon$, is independent of wavenumber $k$. Local cascade also implies that $\epsilon$ is explicitly proportional to $\tau_{3}$ and depends on the wavenumber and on the power of the omni-directional energy spectrum. A simple dimensional analysis leads to

$$
\epsilon=A^{2} \tau_{3}(k) k^{4} E^{2}(k)
$$

where $A$ is a constant. When the time scale for triple decorrelation is simply given by the nonlinear time $\tau_{3}(k)=\tau_{n l}=\left[k^{3 / 2} E^{1 / 2}(k)\right]^{-1}$, the classical Kolmogorov spectrum is recovered.

At asymptotic limits of strong rotation, strong stratification and the limit of strong rotation and stratifcation, there are two disparate time scales. The difference in time scales and anisotropies in length scales are crucial for the mathematical analysis of Babin et al. [6-9] and is the basic requirement for the methodology of our phenomenological analysis (Kraichnan [13], Zhou [14], Mahalov and Zhou [15], Rubinstein and Zhou [5]). The major difficulty encountered in understanding dynamics of geophysical flows is the influence of the oscillations (inertio-gravity waves) generated by the rotation and stratification. This effect leads to the modification of the spectral time for energy transfer down scales.

We recall that the dispersion relation for inertio-gravity waves is given by the formula $\omega_{k}^{2}=N_{0}^{2} \frac{k_{k}^{2}}{k^{2}}+f_{0}^{2} \frac{k_{3}^{2}}{k^{2}}$ where $k=\left(k_{1}, k_{2}, k_{3}\right)$ is the wavevector, $k_{h}^{2}=k_{1}^{2}+k_{2}^{2}$ and $k^{2}=k_{1}^{2}+k_{2}^{2}+k_{3}^{2}$ (axes of rotation and gravity are along the vertical axis $\left.e_{3}=[0,0,1]\right)$. As above, we define the vertical and the horizontal spectral scales 
as $H=\frac{1}{\left|k_{3}\right|}, L=\frac{1}{k_{h}}$. If $a=H / L=k_{h} /\left|k_{3}\right|$ is the ratio of these length scales then $\omega_{k}^{2}=\left(f_{0} / \alpha\right)^{2}+\left(N_{0} / \beta\right)^{2}$ where $\beta=\sqrt{a^{-2}+1}$ and $\alpha=\sqrt{a^{2}+1}$. Then the spectral Burger number, Eq. (3), is a ratio of the spectral stratification frequency $N_{0} / \beta$ and the spectral rotation frequency $f_{0} / \alpha: B u=\frac{\left(N_{0} / \beta\right)^{2}}{\left(f_{0} / \alpha\right)^{2}}=\frac{\left(N_{0} \alpha\right)^{2}}{\left(f_{0} \beta\right)^{2}}=\frac{N_{0}^{2} H^{2}}{f_{0}^{2} L^{2}}$.

We first give a brief treatment for strongly rotating turbulence case. In a regime of high Reynolds numbers and low Rossby numbers, turbulence is characterized by a short time scale $\tau_{f}=\alpha / f_{0}$, where $\alpha=\sqrt{a^{2}+1}$ and $f_{0}=2 \Omega_{0}$. A direct application of $\tau_{3}=\tau_{f}$ results in the energy spectrum for turbulence subject to strong rotation

$$
E(k)=C_{f}\left(\epsilon f_{0} / \alpha\right)^{1 / 2} k^{-2}
$$

where $C_{f}$ is a constant (Zhou [14]). The introduction of the aspect ratio into the time scale is an improvement over our previous phenomenological analysis (Zhou [14], Mahalov and Zhou [15]) since now the model can distinguish the anisotropic nature of rotating flow.

For turbulence in the Burger one regime, the same procedure, namely setting $\tau_{3}=\tau_{f N}$, leads to the energy spectrum for turbulence subject to strong rotation and stratification:

$$
E(k)=C_{f N}\left[\epsilon \sqrt{\left(N_{0} / \beta\right)^{2}+\left(f_{0} / \alpha\right)^{2}}\right]^{1 / 2} k^{-2},
$$

where $\beta=\sqrt{a^{-2}+1}$.

Based on the analogy between the rotating and stratified turbulence (Hopfinger [2]), previous results can be extended to the cases of strongly stratified turbulence. Substituting $\tau_{3}=\tau_{N}$ lcads to

$$
E(k) \sim\left(\epsilon N_{0} / \beta\right)^{1 / 2} k^{-2} .
$$

This reduces to a result found previously by Herring [16] and Kimura and Herring [17] for isotropic case.

We note that, however, based on experimental finding by Dickey and Mellor [18], some further extensions may be needed in the cases of strongly stratified, and probably also for the case of stratified and rotating turbulence. We shall restrict our discussion below to strongly stratified turbulence for brevity. The results can be extended trivially to include the rotation effect by using $\tau_{f N}$ instead of $\tau_{N}$. For a strongly stratified flow, the energy transfer process may be modified in two ways. First, the effect of the internal waves is reflected in the reduced time scales for the triple correlations. This effect reduces the rate of the direct energy transfer down scales and leads to the spectrum predicted by Herring [16]. Second, the effect of the internal waves may lead to a direct reduction in the energy flux, and this reduction is called the "energy radiation rate". Indeed, for moving grid generated turbulence, Dickey and Mellor [18] showed a clear break in the decay rate of the turbulence energy when the buoyancy effects become active. This break indicates the collapse of three dimensional turbulence at nearly all scales. The interpretation given by Dickey and Mellor [18] is that the nonlinear energy transfer now has the general form

$$
\epsilon=\left(u^{3} / l\right)-C N_{0}^{3} l^{2}
$$

Here $u$ is the rms of turbulent velocity, $l$ is the integral scale obtained by integrating the longitudinal velocity autocorrelation, and $C$ is a constant estimated experimentally as $1.910^{-2}$ (Dickey and Mellor [18]).

Including the "energy radiation term" of Dickey and Mellor requires only a very minor modification to our procedure. Noting that Eq. (5) can be rewritten as

$$
\epsilon^{\prime}=A^{2} \tau_{3}(k) k^{4} E^{2}(k)
$$


where we have introduced the effective dissipation rate $\epsilon^{\prime}=\epsilon+C N_{0}^{3} l^{2}$. We remark that when the first effect of stratification is weak $\left(\tau_{3} \sim \tau_{n l}\right)$, the energy spectrum modified by the second effect of stratification takes the form

$$
E(k) \sim\left(\epsilon^{\prime}\right)^{2 / 3} k^{-5 / 3}
$$

In general, the lifetime of triple correlations in rotating and stratified turbulence might be more accurately treated by taking into account the possibility that these correlations decay because of the influence of both wave propagation and nonlinear triadic interactions (Zhou [14]). The simple choice

$$
\frac{1}{\tau_{3}(k)}=\frac{1}{\tau_{n l}(k)}+\frac{1}{\tau_{E}(k)}
$$

satisfies the appropriate limiting cases: $\tau_{3}(k) \rightarrow \tau_{n l}$ without external agencies, and $\tau_{3}(k) \rightarrow \tau_{E}$ with external agencies. Here $\tau_{E}$ may be taken as $\tau_{f}, \tau_{N}$, or $\tau_{f N}$.

We now find that the general energy spectrum for strongly stratified turbulence takes the form

$$
E(k)=Z^{2} A^{-4 / 3} \epsilon^{\prime 2 / 3} k^{-5 / 3},
$$

where $Z^{\prime}$ is given by

$$
Z^{\prime}=\frac{1}{2}\left(\sqrt{Y}^{\prime}+\sqrt{-Y^{\prime}+2 \sqrt{Y^{\prime 2}+4 Z_{0}^{\prime}}}\right)
$$

where

$$
Y^{\prime}=\sqrt[3]{\frac{1}{2}+\sqrt{\frac{1}{4}+\left(\frac{4 Z_{0}^{\prime}}{3}\right)^{3}}}+\sqrt[3]{\frac{1}{2}-\sqrt{\frac{1}{4}+\left(\frac{4 Z_{0}^{\prime}}{3}\right)^{3}}}
$$

The parameters $A=C_{K}^{-3 / 4}$, and $Z_{0}^{\prime}=\left[\frac{A k_{E}^{\prime}}{k}\right]^{2 / 3}$. Again, depending on the situation, $k_{E}^{\prime}$ may take values from $k_{N}^{\prime}=\left(N_{0}^{3} / \epsilon^{\prime}\right)^{1 / 2}$ (for strongly stratified flows), and $k_{f N}^{\prime}=\left(\left[\left(N_{0} / \beta\right)^{2}+\left(f_{0} / \alpha\right)^{2}\right]^{3 / 2} / \epsilon^{\prime}\right)^{1 / 2}$ (for stratified/rotating flows). These equations reduce to the classical Kolmogorov " $-5 / 3$ " spectrum when $Z_{0}^{\prime} \rightarrow 0$ (so that $Z^{\prime} \rightarrow 1$ ), and to our strongly stratified or rotation/stratification modified "-2" spectrum when $Z_{0}^{\prime} \rightarrow \infty$ (so that $Z^{\prime} \rightarrow Z_{0}^{\prime 1 / 4}$ ). Alternatively, the scaling of the energy spectrum in strongly stratificd case may remain as $-5 / 3$ (as pointed out already, by $\tau_{3} \sim \tau_{n l}$ ). For intermediate strength of the stratification (or rotation/stratification) the spectrum varies smoothly between these two limiting forms, according to the increase of the controlling parameter $Z_{0}^{\prime}$ with increasing ratio $k_{E}^{\prime} / k$.

We note the difference between the energy spectra in a 2D case (Kraichnan [19]) and quasi-2D case. Based on statistical turbulence theory, Herring [16] find that it is quite difficult to justify a $2 \mathrm{D}$ spectra $-5 / 3$ (for scales larger than the energy injection scale) and -3 spectra (for scales smaller than the energy injection scale) for a stratified flow. The reason is that the triple-moment relaxation is dominated by waves. The fact that the time scale for triple velocity correlation is dominanted by faster time scale forms the foundation for our development and the work by Herring [16].

3. Conclusions. We conclude this note by noting that our energy spectrum, in its most general form, would lead to an energy spectrum which changes smoothly between the Kolmogorov $-5 / 3$ law (no stratification), the -2 scalings of Zhou [14] for the case of strong rotation, as well as the -2 scalings for the case of both strong rotation and stratification. For strongly stratified turbulence, the model may give the -2 scaling predicted by Herring [16] and the $-5 / 3$ scaling indicated by some observations at mesoscales. 


\section{REFERENCES}

[1] Pedlosky, J., Geophysical Fluid Dynamics, 2nd edition, Springer-Verlag, 1987.

[2] Hopfinger, E.J., Turbulence in stratified fuids: A review, J. Geophys. Res., 92, 1987, p. 5287.

[3] RoDI, W., Examples of calculation methods for flow and mixing in stratified fluids, J. Geophys. Res., 92, 1987, p. 5305.

[4] Fernando, H.J.S. And Hunt, J.C.R., Some aspects of turbulence and mixing in stably stratified layers, Dyn. of Atm. and Oceans, 23, 1996, p. 35.

[5] Rubinstein, R. And Zhou, Y., Analytical theory of the destruction terms in dissipation rate transport equations, Physics of Fluids, 8, 1996, p. 7172,

[6] Babin, A., Mahalov, A., ANd Nicolaenko, B., Global splitting, integrability and regularity of $3 D$ Euler and Navier-Stokes equations for uniformly rotating fluids, Europ. J. of Mech., B/Fluids, 15, 1996, p. 291.

[7] Babin, A., Mahalov, A., AND Nicolaenko, B., Resonances and regularity for Boussinesq equations, Russian J. Math. Phys., 4, 1996, p. 417.

[8] Babin, A., Mahalov, A., Nicolaenko, B., And Zhov, Y., On the asymptotic regimes and the strongly stratified limit of Boussinesq equations, Theor. and Comput. Fluid Dyn., (in press), 1997.

[9] Babin, A., Mahalov, A., AND Nicolaenko, B., Global regularity and integrability of $3 D$ Euler and Navier-Stokes equations for rotating fluids, Asympt. Anal., (in press), 1997.

[10] MCWILLIAMS, J.C., A note on a uniformly valid model spanning the regimes of geostrophic and isotropic stratified turbulence: Balanced turbulence, J. Atmos. Sci., 42, 1985, p. 1773.

[11] Lesieur, M., Turbulence in Fluids, Kluwer Academic Press, 1990.

[12] Farge, M. And Sadourny, R., Wave-vortex dynamics ir rotating shallow layer, J. Fluid Mech., 206, 1989, p. 433.

[13] KRAICHNAN, R.H., Inertial range spectrum of hydromagnetic turbulence, Phys. Fluids, 8, 1965, p. 1385.

[14] ZHov, Y., A phenomenological treatment of rotating turbulence, Phys. Fluids, 7, 1995, p. 2092.

[15] Mahalov, A. AND ZhOU, Y., Analytical and phenomenological studies of rotating turbulence, Phys. of Fluids, 8, 1996, p. 2138.

[16] Herring, J.R., The inverse cascade range of quasi-geostrophic turbulence, Metcorol. Atmos. Phys., 38, 1988, p. 106.

[17] Kimura, Y. And Herring, J.R., Diffusion in stably strutified turbulence, J. Fluid Mech., 328, 1996, p. 253.

[18] DickeY, T.D. AND MELloR, G.L., Decaying turbulence in neutral and stratified fluids, J. Fluid Mech., 99, 1980, p. 13.

[19] KRAiChnan, R.H. Inertial range in two-dimensional turbulence, Phys. of Fluids, 10, 1967, p. 1417. 



\begin{tabular}{|c|c|c|c|}
\hline \multicolumn{3}{|c|}{ REPORT DOCUMENTATION PAGE } & $\begin{array}{l}\text { Form Approved } \\
\text { OMB No. } 07040188\end{array}$ \\
\hline \multicolumn{4}{|c|}{$\begin{array}{l}\text { Public reporting burden for this collection of information is estimated to average } 1 \text { hour per response, including the time for reviewing instructions, searching existing data sources, } \\
\text { gathering and maintaining the data needed, and completing and reviewing the collection of information. Send comments regarding this burden estimate or any orher aspect of this } \\
\text { collection of information, including suggestions for reducing this burden, to Washington Headquarters Services, Directorate for Information Operations and Reports. } 1215 \text { Jefferson } \\
\text { Davis Highway. Suite 1204. Arlington, VA 22202-4302, and to the Office of Management and Budget, Paperwork Reduction Project (0704-0188). Washington. DC } 20503 \text {. }\end{array}$} \\
\hline 1. AGENCY USE ONLY(Leave blank) & \begin{tabular}{|l|}
$\begin{array}{l}\text { 2. REPORT DATE } \\
\text { July } 1998\end{array}$ \\
\end{tabular} & \multicolumn{2}{|c|}{$\begin{array}{l}\text { 3. REPORT TYPE AND DATES COVERED } \\
\text { Contractor Report }\end{array}$} \\
\hline \multicolumn{3}{|c|}{$\begin{array}{l}\text { 4. TITLE AND SUBTITLE } \\
\text { Energy spectra of strongly stratified and rotating turbulence }\end{array}$} & \multirow[t]{2}{*}{$\begin{array}{l}\text { 5. FUNDING NUMBERS } \\
\text { C NAS1-97046 } \\
\text { WU 505-90-52-01 }\end{array}$} \\
\hline \multicolumn{3}{|c|}{$\begin{array}{l}\text { 6. AUTHOR(S) } \\
\text { Alex Mahalov } \\
\text { Basil Nicolaenko } \\
\text { Ye Zhou } \\
\end{array}$} & \\
\hline \multicolumn{3}{|c|}{$\begin{array}{l}\text { 7. PERFORMING ORGANIZATION NAME(S) AND ADDRESS(ES) } \\
\text { Institute for Computer Applications in Science and Engineering } \\
\text { Mail Stop 403, NASA Langley Research Center } \\
\text { Hampton, VA 23681-2199 }\end{array}$} & $\begin{array}{l}\text { 8. PERFORMING ORGANIZATION } \\
\text { REPORT NUMBER } \\
\text { ICASE Report No. } 98-28\end{array}$ \\
\hline \multicolumn{3}{|c|}{$\begin{array}{l}\text { 9. SPONSORING/MONITORING AGENCY NAME(S) AND ADDRESS(ES) } \\
\text { National Aeronautics and Space Administration } \\
\text { Langley Research Center } \\
\text { Hampton, VA 23681-2199 }\end{array}$} & $\begin{array}{l}\text { 10. SPONSORING/MONITORING } \\
\text { AGENCY REPORT NUMBER } \\
\text { NASA/CR-1998-208448 } \\
\text { ICASE Report No. } 98-28\end{array}$ \\
\hline \multicolumn{4}{|c|}{$\begin{array}{l}\text { 11. SUPPLEMENTARY NOTES } \\
\text { Langley Technical Monitor: Dennis M. Bushnell } \\
\text { Final Report } \\
\text { To appear in Physical Review E }\end{array}$} \\
\hline \multicolumn{2}{|c|}{$\begin{array}{l}\text { 12a. DISTRIBUTION/AVAILABILITY STATEMENT } \\
\text { Unclassified Unlimited } \\
\text { Subject Category } 34 \\
\text { Distribution: Nonstandard } \\
\text { Availability: NASA-CASI (301)621-0390 }\end{array}$} & & 12b. DISTRIBUTION CODE \\
\hline \multicolumn{4}{|c|}{$\begin{array}{l}\text { 13. ABSTRACT (Maximum } 200 \text { words) } \\
\text { Turbulence under strong stratification and rotation is usually characterized as quasi-two dimensional turbulence. } \\
\text { We develop a "quasi-two dimensional" energy spectrum which changes smoothly between the Kolmogorov }-5 / 3 \text { law } \\
\text { (no stratification), the }-2 \text { scalings of Zhou for the case of strong rotation, as well as the }-2 \text { scalings for the case of } \\
\text { strong rotation and stratification. For strongly stratified turbulence, the model may give the }-2 \text { scaling predicted by } \\
\text { Herring; and the }-5 / 3 \text { scaling indicated by some mesoscale observations. }\end{array}$} \\
\hline \multirow{2}{*}{\multicolumn{2}{|c|}{$\begin{array}{l}\text { 14. SUBJECT TERMS } \\
\text { stratification; rotation; turbulence modeling }\end{array}$}} & 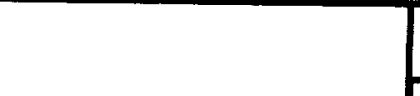 & $\begin{array}{l}\text { 15. NUMBER OF PAGES } \\
11 \\
\end{array}$ \\
\hline & & 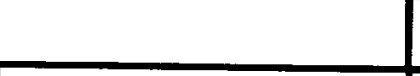 & $\begin{array}{l}\text { 16. PRICE CODE } \\
\text { A02 } \\
\end{array}$ \\
\hline $\begin{array}{l}\text { 17. SECURITY CLASSIFICATION } \\
\text { OF REPORT } \\
\text { Unclassified }\end{array}$ & $\begin{array}{l}\text { 18. SECURITY CLASSIFICATION } \\
\text { OF THIS PAGE } \\
\text { Unclassified }\end{array}$ & $\begin{array}{l}\text { 19. SECURITY CLASSIFICATION } \\
\text { OF ABSTRACT }\end{array}$ & $\begin{array}{l}\text { 20. LIMITATION } \\
\text { OF ABSTRACT }\end{array}$ \\
\hline
\end{tabular}

